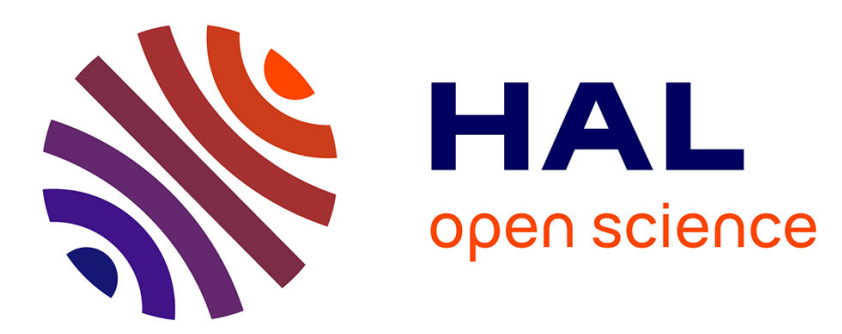

\title{
Cholesteric microlenses and micromirrors in the beetle cuticle and in synthetic oligomer films: a comparative study
}

Gonzague Agez, Chloé Bayon, Michel Mitov

\section{- To cite this version:}

Gonzague Agez, Chloé Bayon, Michel Mitov. Cholesteric microlenses and micromirrors in the beetle cuticle and in synthetic oligomer films: a comparative study. SPIE OPTO, Jan 2017, San Francisco, United States. pp.101250U, 10.1117/12.2260752 . hal-01730529

\section{HAL Id: hal-01730529 \\ https://hal.science/hal-01730529}

Submitted on 12 Feb 2021

HAL is a multi-disciplinary open access archive for the deposit and dissemination of scientific research documents, whether they are published or not. The documents may come from teaching and research institutions in France or abroad, or from public or private research centers.
L'archive ouverte pluridisciplinaire HAL, est destinée au dépôt et à la diffusion de documents scientifiques de niveau recherche, publiés ou non, émanant des établissements d'enseignement et de recherche français ou étrangers, des laboratoires publics ou privés. 


\title{
Cholesteric microlenses and micromirrors in the beetle cuticle and in synthetic oligomer films: a comparative study
}

\author{
Gonzague Agez, Chloé Bayon, Michel Mitov* \\ Centre d'Elaboration de Matériaux et d'Etudes Structurales, CEMES, CNRS, \\ University Paul-Sabatier, 29 rue Jeanne-Marvig, BP 94347, F-31055 Toulouse cedex 4, France
}

\begin{abstract}
The polygonal texture in cholesteric liquid crystals consist in an array of contiguous polygonal cells. The optical response and the structure of polygonal texture are investigated in the cuticle of beetle Chrysina gloriosa and in synthetic oligomer films. In the insect carapace, the polygons are concave and behave as spherical micro-mirrors whereas they are convex and behave as diverging microlenses in synthetic films. The characteristics of light focusing (spot, donut or continuum background) are highly tunable with the wavelength and the polarization of the incident light.
\end{abstract}

Keywords: cholesteric liquid crystals, beetle cuticle, microlenses, micromirrors, structural color, bio-inspiration.

\section{INTRODUCTION}

Microlenses concern a variety of applications including the coupling of light from optical fibres and LEDs, the improvement of the optical efficiency of CCDs for cameras, LCDs for projectors and flat panel displays, and the generation of 3D images ${ }^{1,2}$. Micromirrors are used in optical devices, tunable lasers ${ }^{3}$, televisions, hard disk storage, projectors, imaging technologies ${ }^{4}$ and MEMS technology for medical applications ${ }^{5}$ or optical bio-imaging ${ }^{6}$.

The purpose of the present investigation is to provide a comparison of optical behaviors and structures from microlenses found in synthetic cholesteric liquid crystal (CLC) oligomer films ${ }^{7,8}$ to cholesteric micro-mirrors in the cuticle (outer carapace) of the beetle Chrysina gloriosa (C. gloriosa $)^{9}$.

\section{OPTICAL TEXTURES}

The cuticle of $C$. gloriosa exhibits green and silver stripes (Fig. 1.a). Fig. 2.b shows the interface between stripes. The green stripe is a mosaic of contiguous polygonal cells. Although the transition between stripes is sharp, a few partially formed polygons are dispersed in the silver stripe close to the frontier zones. Each polygonal cell contains a bright yellow core with orange edges surrounded by a dark-green region, with a brighter green rim delimiting the cells. In the following we only address the green stripe, which contains micromirrors.

CLC oligomers from Wacker Chemie $\mathrm{GmbH}$ are used ${ }^{10}$. The CLC phase appears between $180-210^{\circ} \mathrm{C}$ (clearing temperature range) and $40-50^{\circ} \mathrm{C}$ (glass-transition temperature range). CLC films may be coated on a flexible plastic foil allowing curvature radii close the curvature of the cuticle (Fig. 1.c). In the present study, 13- $\mu \mathrm{m}$ thick films are formed at $140^{\circ} \mathrm{C}$ between two glass plates. The sandwich-cell is promptly quenched at room temperature (RT). The upper plate is removed after quenching below the glass transition temperature, when the film is solid as a vitrified CLC. The annealing of the open film occurs at $140^{\circ} \mathrm{C}$ for 5 hours, which time corresponds to the formation of an array of microlenses with an optimized focal point (clear-cut shape and maximum transmitted intensity). The open film is finally rapidly quenched at RT. The spontaneous nucleation and growth of the polygonal texture gives rise to an array of polygons with various sizes as shown in Fig. 1.d. Each polygonal cell contains a black dot surrounded by a yellow/orange region with a brighter yellow rim delimiting the cells.

\footnotetext{
*mitov@cemes.fr; phone +33562257861; fax +33562257999; cemes.fr
} 

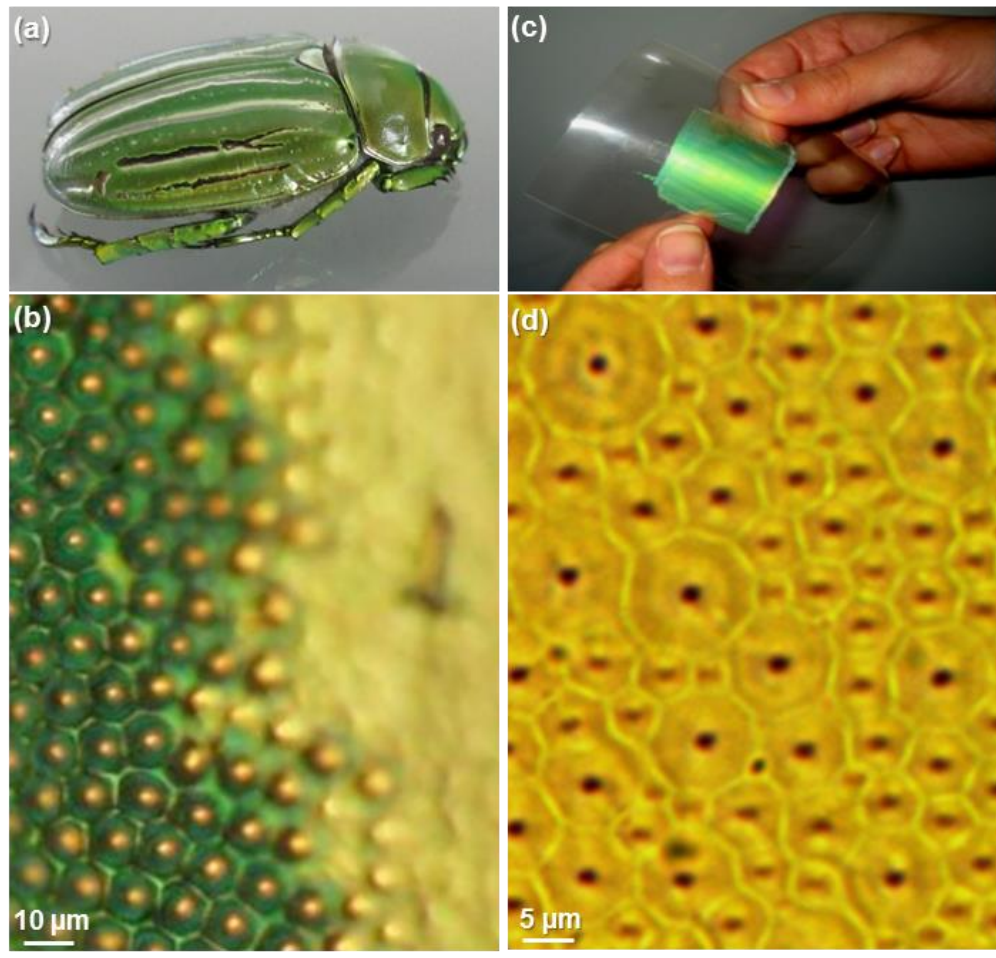

Figure 1. Materials and optical textures. (a) Beetle Chrysina gloriosa. (b) Interface between green and silver stripes (reflection mode, unpolarized light). (c) Cholesteric oligomer film coated on a curved flexible substrate. (d) Polygonal cholesteric texture of the oligomer film (transmission mode, unpolarized light).

\section{TRANSMISSION ELECTRON MICROSCOPY STUDY}

90-nm thick cross-sectional cuts of cuticle and oligomer film are obtained by using an ultramicrotome (UltraCut S from Reichert) equipped with a diamond knife. They are observed by transmission electron microscopy (TEM; Philips CM20 FEG microscope operating at $200 \mathrm{kV}$ in Fig. 2.a; CM30 microscope operating at $300 \mathrm{kV}$ in Fig. 2.b). In both cases a typical fingerprint texture is displayed. The distance between two lines of equal contrast is related to the pitch of the twisted structure, while the helicoidal axis is perpendicular to the lines. In the case of the cuticle (Fig. 2.a), the typical fingerprint texture is displayed in the chitin network of the cuticle and presents concave nested arcs below each polygon. At the air-cuticle interface, wax fills the cavity of the polygonal cells. At the surface of the cuticle, a crack is very often visible in the wax at the top of a polygon (Fig. 3). We use this kind of artifact to localize precisely the position of the polygon with regard to the transversal structure (Fig. 3), and to report in Fig. 2.a the so-named optical axis. In the case of the oligomer film (Fig. 2.b), the air interface (resp. glass plate interface) is at the top (resp. bottom) of the image. The fingerprint texture presents convex nested arcs below each polygon of the oligomer film. We have reported for a couple of polygons the optical axis which intercepts the top of polygonal cells. The cuticle surface is quite flat due to the presence of the wax layer which fills the pits in concave mirrors whereas the convex polygons in the synthetic film exhibits a small relief in the $20-100 \mathrm{~nm}$ range as shown by $\mathrm{AFM}^{11}$.

Sharma et al. ${ }^{12}$ described the polygonal cells of $C$. gloriosa as completely analogous to the focal conic domains found in open oligomer films used in Ref. [13]. Agez et al. ${ }^{11}$ and Bayon et al. ${ }^{7}$ have mentioned analogies between the polygonal textures found in the $C$. gloriosa cuticle and in the same open oligomer films. The present investigation highlights fundamental differences. The most important differentiator is that the nested arced patterns inside a cholesteric polygon are convex for the synthetic film while they are concave for the biological material. Besides, the synthetic film presents no pitch gradient when a pitch gradient from visible to NIR spectrum is present in the cuticle 9 . 


\section{Invited Paper}
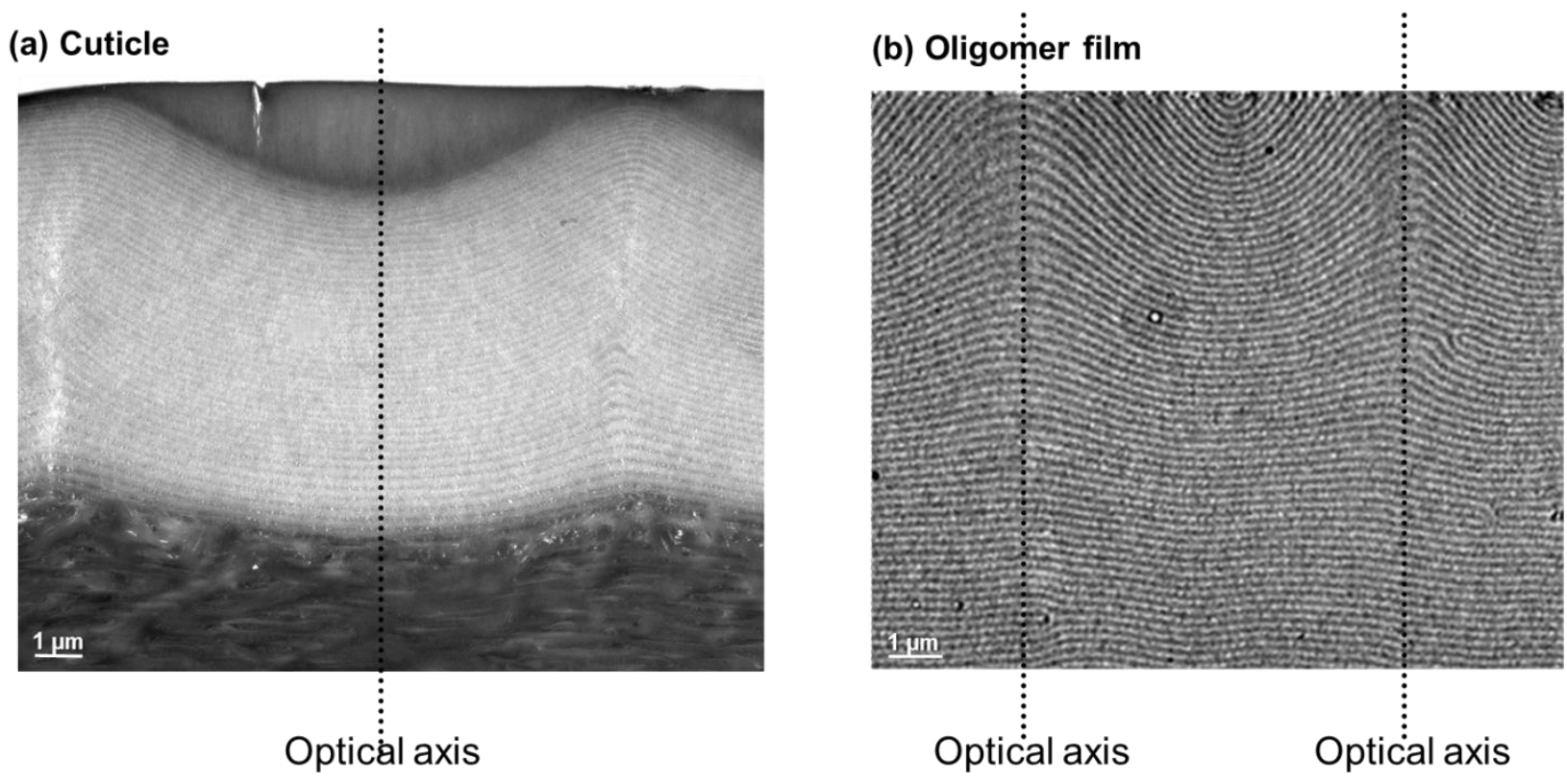

Figure 2. Transverse TEM views of the beetle cuticle and the oligomer film.

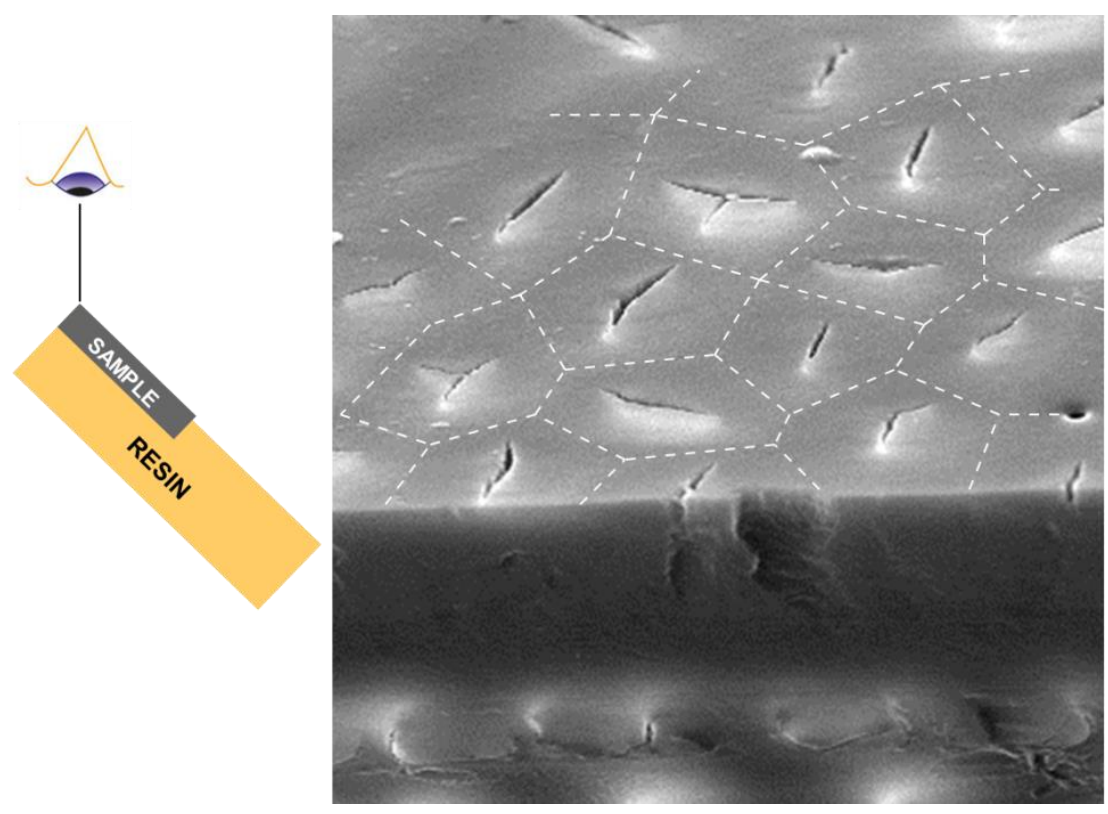

Figure 3. Scanning electron microscopy perspective view of the cuticle showing the correspondence between the polygons with cracks in the cuticle plane and the transverse section. 


\section{MAPPING OF REFLECTED INTENSITY}

We used a spectrometer equipped with a standard confocal microscope (XploRA from Horiba) to scan the layer over the area of a single polygon at RT. The investigation of the light transmitted through the oligomer film (Fig. 4.a) shows that the light focusing is controlled with the wavelength of the incident light and typically corresponds to a spot in the 400$460 \mathrm{~nm}$ range and to a donut in the $600-800 \mathrm{~nm}$ range. The microlenses are diverging ${ }^{7}$. The intrinsic helical organization of the layer is the cause of the light focusing and not the shape of the surface as for conventional lenses. We name these microlenses as Bragg microlenses.

\section{(a) Oligomer film}
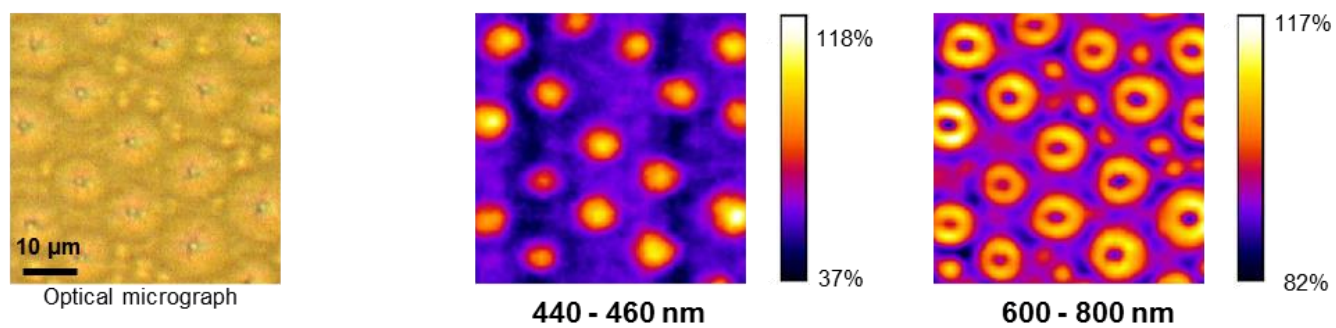

\section{(b) Cuticle}

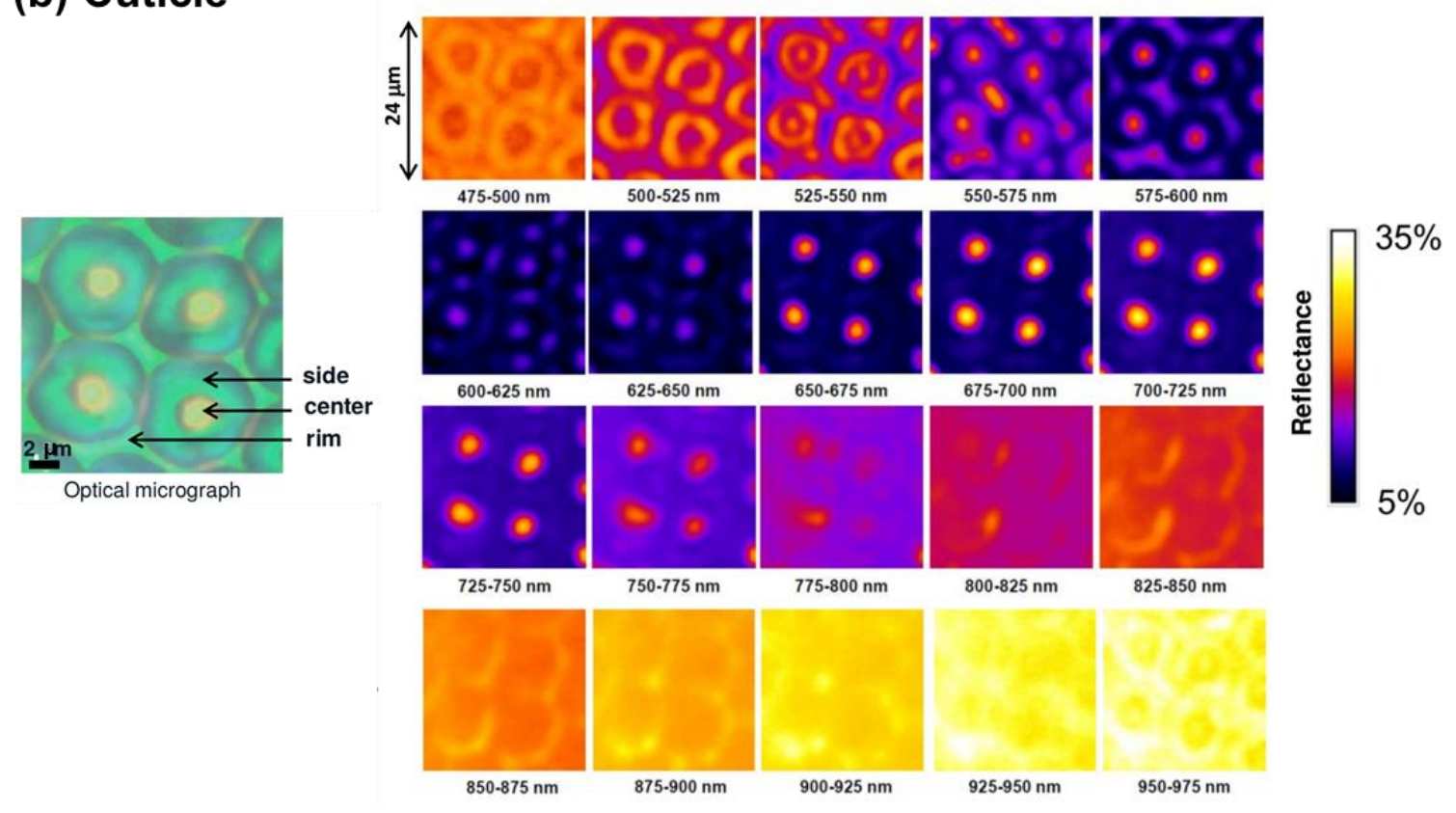

Figure 4. (a) Mapping of the light transmitted through the oligomer film (annealed at $140^{\circ} \mathrm{C}$ for 5 hours) in the film plane. Two spectral bands of interest were chosen inside the Bragg band $(440-460 \mathrm{~nm})$ and inside the red band $(600-800 \mathrm{~nm})$. The scale bar depicts the 8-bit lookup table used for color representation and refers to the percentage of transmission per area unit. The scales are

different to facilitate the representation. The baseline is recorded with the glass substrate only. Since the light is differently concentrated on the polygon surface, the transmission scale can reach values above $100 \%$. (b) Mapping of light reflected by the cuticle at the focus plane for twenty wavelength ranges. $100 \%$ of reflection corresponds to a flat silver mirror. 
In both cases, an optical micrograph of the investigated region is inserted.

The mapping of light reflected by the cuticle for various wavelengths, from blue (475 nm) to NIR (975 nm), with a narrow wavelength increment of $25 \mathrm{~nm}$, is shown in Fig. 4.b. The microscope focus was set to the air-wax interface. Intense reflections occur along the sides of the polygons and manifest as donut-like patterns from 475-500 $\mathrm{nm}$ to 525 $550 \mathrm{~nm}$. The donuts extinguish in the 550-575 nm map. After transitional patterns into the next three spectral regions, a bright central spot - at the intersection between the focus plane and the optical axis as marked in Fig. 2.a-appears in the 650-675 nm range. This spot corresponds to the polygon center and the reflection intensifies into the next two channels, up to $725 \mathrm{~nm}$. The reflection of spots then decreases from the 725-750 nm range. After transitional patterns in between visible and NIR spectra, from 750 to $825 \mathrm{~nm}$, reflection patterns occurring in rims remain visible until the end of the investigation at $975 \mathrm{~nm}$. The main feature of this sequence (from $825 \mathrm{~nm}$ ) is that the reflection continuously increases as the spectrum moves into the NIR.

\section{POLARIZATION EFFECTS}

The mapping of the light transmitted by the oligomer film is investigated in the red band (600-800 nm) in the presence of a linear analyzer with different orientations (Fig. 5.a), when the incoming light is unpolarized. The light focuses like a pair of crescents which rotate with the orientation of the analyzer. The radial polarization state of the donut is highlighted. The mapping of transmitted light is then investigated in the Bragg band (470-500 nm) (Fig. 5.b). Since the CLC helix of the oligomer film is left-handed, when the incident light is right-handed circularly polarized (RHCP) or left-handed (LH) CP, the existence of a brilliant spot in the former case and the very low reflected intensity in the latter one shows the spatial distribution of the CP light. The polygonal cell represents a complex situation since the orientation of the helicoidal axis is continuously changing inside the volume of a cell, so the polarization-selectivity rule ${ }^{14,15}$ does not strictly apply because it is valid at only normal incidence, when the direction of light propagation and the helicoidal axis coincide. At oblique incidence the reflected or transmitted light is elliptically polarized.

(a) Red band (600-800) nm
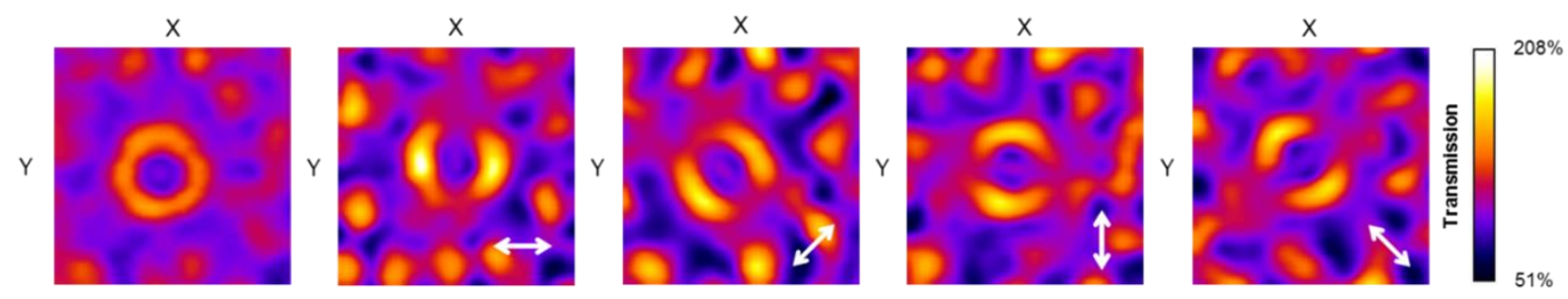

(b) Bragg band (470-500) $\mathrm{nm}$
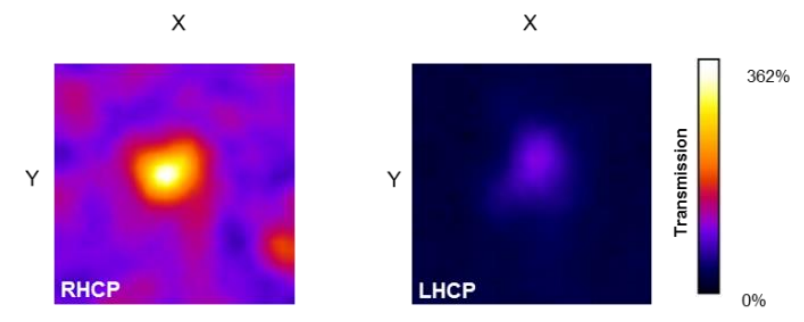

Figure 5. (a) In the red band: mapping of light transmitted by the oligomer film without analyzer (first map) and in the presence of a linear analyzer with different orientations (white double arrow). (b) In the Bragg band: mapping of light transmitted by the oligomer film when the incident light is right-handed and left-handed circularly polarized. 
Figure 6 represents an image of beetle C. gloriosa, with the corresponding microtextures, when a LH or a RH circular polarizer is inserted between the beetle and the observer. The incident light is unpolarized. Because the CLC helix of the cuticle is here also left-handed, the beetle and the polygons appear as very brilliant in the former case and very dark in the latter case. Contiguous regions are shown in Fig. 6.b; a few polygons at the border area have been enhanced in black. The cracks related to polygons are revealed in the presence of a $\mathrm{RH}$ circular polarizer.

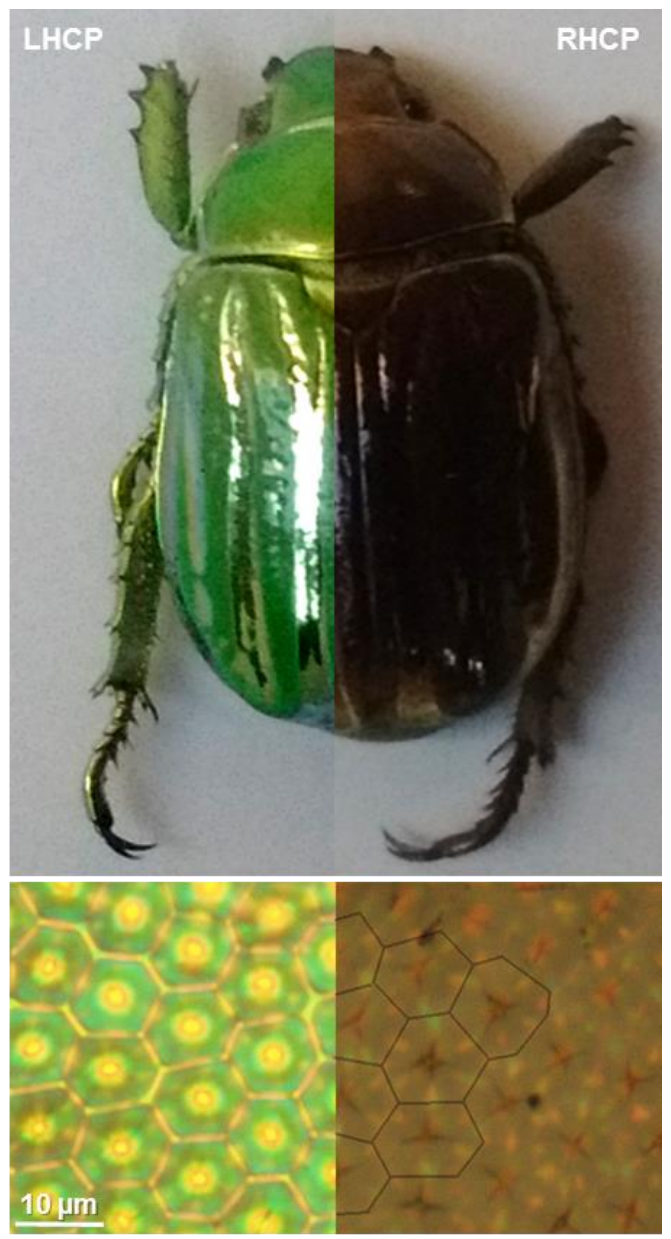

Figure 6. Macroscopic view of the beetle and microtextures of the cuticle when a left-handed (LHCP) or right-handed circular polarizer (RHCP) is inserted between the beetle and the observer.

\section{CONCLUSIONS}

In oligomer films, the structure and the light transmission properties of microlenses were characterized and it was shown that their focusing properties, as a spot or as a donut, were wavelength-tunable. To provide the focusing effect, CLC microlenses rely on the intrinsic chiral organization of CLC and not on the shape of the lens as usually. Related applications are in the field of wavelength-tunable chiro-optical devices and lab-on-a-chip optical systems that offer the combined benefits of multiple light manipulation capabilities, seamless integration and mechanical stability. In the cuticle: (i) the reflectance is spatially sensitive to wavelength changes, the highest levels of reflectance come from different parts of a polygon-from the sides in the lower end of the visible spectrum and longer wavelengths from the 
center; (ii) in the NIR spectrum, from 825 to $975 \mathrm{~nm}$, the variation in reflectance consists of a mean level change weakly sensitive to the patterning. This result is consistent with the structure since the cholesteric pseudo-layers in the endocuticle, which generate the NIR reflections, appear continuously parallel ${ }^{9}$; the orientation of the helicoidal axis changes slightly and this deeper part of the cuticle is not sharply tessellated, and thus the reflection pattern is weakly structured. The reflection properties of the polygon arrays in the green stripes of $C$. gloriosa cuticles may serve as an inspiration for the fabrication of wavelength-tunable micromirrors. Each micromirror could serve as a wavelengthselective spatial light modulator, optimized for small form-factor applications and with highly flexible light patterns. Such micromirrors are desirable for fiber optics or optical switching, where micromirror technology is essential for increasing the speed of communication.

\section{Acknowledgements}

The authors thank Dr. E. Hanelt from Wacker-Chemie GmbH (Munich, Germany) for providing them with oligomers and constant support, and Dr. A. Zwick (CEMES) for his technical assistance with XploRA.

\section{REFERENCES}

[1] Stevens R. and Miyashita T., "Review of standards for microlenses and microlens arrays", Imaging Sci. J., 58, 202-212 (2010).

[2] Hou T., Zheng C., Bai S., Ma Q., Bridges D., Hu A. and Duley W. W., "Fabrication, characterization, and applications of Microlenses", Appl. Optics, 54, 7366-7376 (2015).

[3] Liu, A.Q.; Zhang, X.M., "A review of MEMS external-cavity tunable lasers”, J. Micromech. Microeng., 17, R1R13 (2007).

[4] Dudley, D., Duncan, W.M. and Slaughter, J., "Emerging digital micromirror device (DMD) applications", Proc. SPIE, 4985, 14-25 (2003).

[5] Pengwang E., Rabenorosoa K., Rakotondrabe M. and Andreff N., "Scanning Micromirror Platform Based on MEMS Technology for Medical Application”, Micromachines, 7, 1-29 (2016).

[6] Lin L. Y. and Keeler E. G., "Progress of MEMS Scanning Micromirrors for Optical Bio-Imaging", Micromachines, 6, 1675-1689 (2015).

[7] Bayon C., Agez G. and Mitov M., "Wavelength-tunable light shaping with cholesteric liquid crystal microlenses", Lab Chip, 14, 2063-2071 (2014).

[8] Bayon C., Agez G. and Mitov M., "Size-effect of oligomeric cholesteric liquid-crystal microlenses on the optical specifications", Optics Lett., 40, 4763-4766 (2015).

[9] Agez G., Bayon C. and Mitov M., "Multiwavelength micromirrors in the cuticle of scarab beetle Chrysina gloriosa", Acta Biomater., 48, 357-367 (2017).

[10] Agez G. and Mitov M., "Cholesteric liquid crystalline materials with a dual circularly polarized light reflection band fixed at room temperature", J. Phys. Chem. B 115, 6421 (2011).

[11] Agez G., Bitar R., Mitov M., "Color selectivity lent to a cholesteric liquid crystal by monitoring interface-induced deformations", Soft Matter, 7, 2841-284(2011).

[12] Sharma V., Crne M., Park J. O. and Srinivasarao M., "Structural origin of circularly polarized iridescence in jeweled beetles", Science, 325, 449-451 (2009).

[13] Meister R., Dumoulin H., Hallé M.-A. and Pieranski P., "The anchoring of a cholesteric liquid crystal at the free surface”, J. Phys. II France, 6, 827-844 (1996).

[14] The reflected light is CP with the same handedness that of the CLC structure.

[15] Kelker H. and R. Hatz R., [Handbook of Liquid Crystals], Verlag Chemie, Weinheim, Germany, 293-337 (1980). 